\title{
ENSAYO SOBRE UNA NUEVA CLASIFICACIÓN \\ DE POBLACIÓN RURAL Y URBANA \\ EN MÉXICO
}

\author{
LUIS UNIKEL * \\ El Colegio de México
}

PARA PODER describir el proceso de urbanización desde un punto de vista demográfico-ecológico, es necesario contar con una clasificación de población rural y urbana. ${ }^{1}$ El objeto de este trabajo es determinar una clasificación que, al mismo tiempo que sea manejable estadísticamente, se base en fundamentos teóricos y metodológicos que reduzcan tanto las deficiencias de los criterios censales y no censales que se aplican en el país como, en general, el grado de arbitrariedad implícito en toda clasificación de población de este tipo.

El trabajo se divide en dos partes. En la primera se exponen brevemente los principales enfoques teóricos de las distintas clasificaciones de población urbana y rural, algunas consideraciones sobre las mismas, y diversos criterios censales y no censales aplicados para el establecimiento de distinciones urbano-rurales. Con ello se pretende, además de sentar las bases para el desarrollo del análisis, dar a conocer algunos elementos que faciliten la realización de estudios más profundos sobre la definición de lo urbano y lo rural en México.

En la segunda parte se determina una nueva clasificación de la población urbana y rural que constituya un instrumento operativo cuya aplicación permita acercarse al conocimiento de la realidad nacional y regional del desarrollo urbano en México. Asimismo, se espera que el trabajo sirva de estímulo para la elaboración de estudios semejantes en otros países, especialmente en América Latina, de tal modo que las clasificaciones resultantes faciliten la realización de investigaciones comparativas sobre el desarrollo urbano.

* El autor agradece a Julio Boltvinik, Federico Torres y Edmundo Victoria, del Centro de Estudios Económicos y Demográficos, la colaboración prestada en este trabajo. Asimismo está reconocido a otros investigadores del Centro que aportaron sugestiones y comentarios a una versión preliminar del estudio.

$1 \mathrm{El}$ proceso de urbanización, desde un punto de vista demográfico-ecológico, se define como la concentración de la población por la cual aumenta la proporción de la población urbana con relación a la total, a través del crecimiento de las localidades urbanas existentes y de la multiplicación de los puntos geográficos de concentración demográfica. Véanse Jaime Dorselaer y Alfonso Gregory, La urbanización en América Latina, Bogotá, Feres, tomo I, p. 11; Hope T. Eldridge, "The Process of Urbanization", en Joseph Spengler y Otis Duncan (compiladores), Demographic Analysis, Nueva York, The Free Press of Glencoe, 1963, pp. 338-343. 


\section{ENFOQUES TEÓRICOS PARA DIFERENCIAR A LA POBLACIÓN URBANA Y RURAL}

"A primera vista, la definición de población urbana y rural parece ser evidente: población urbana es toda aquella que vive en las ciudades, y rural la que no vive en ciudades." 2 Sin embargo, el problema se complica considerablemente cuando surge la pregunta de lo que es una ciudad, o de cuáles son las características básicas que identifican a la sociedad urbana en contraste con la no urbana. La dificultad para contestar tales preguntas se hace patente en la vaguedad de la terminología hasta hoy empleada en las definiciones, ${ }^{3}$ así como en la variedad de las mismas. La ciudad ha sido definida según criterios demográficos, ecológicos, históricos, jurídicos, económicos, sociológicos, etc., 4 multiplicidad de definiciones que se explica en parte por la variedad de intereses disciplinarios, pero fundamentalmente por la complejidad de la naturaleza de la ciudad, que es imposible englobar en una sola definición válida para todo tipo de sociedad y para cualquier época.

El análisis y la descripción de las características urbanas y no urbanas de una sociedad, desde el punto de vista de la sociología urbana, se han hecho con base en tres enfoques generales: a) el de la comunidad de tipo ideal (ideal-type community approach); b) el del continuum rural-urbano, y c) el del conjunto de características (traitcomplex approach).

Enfoque de la comunidad de tipo ideal. El primer enfoque plantea la existencia de dos tipos de sociedades, una tradicional (folk) y otra urbana, cada una caracterizada por una serie de abstracciones lógicas, absolutas y excluyentes, que conducen a la formación de un esquema teórico correspondiente a una sociedad dicotómica. Redfield definió a la sociedad folk como una sociedad "pequeña, aislada, analfabeta, homogénea, cuyos miembros tienen gran sentido de solidaridad de grupo" 5 y, por lo tanto, atribuyó a la sociedad urbana características opuestas. L. Wirth define la ciudad como "una aglomeración permanente relativamente grande y densa de individuos socialmente heterogéneos". 6

La mayoría de los análisis de las poblaciones urbana y rural ha sido de índole dicotómica, lo cual se ha debido principalmente a lo atractivo de su simplicidad. Pero esto se hace mecánicamente sin reparar en el significado que tiene en la realidad. Así lo señala una

2 Dorselaer y Gregory, op. cit., p. 13.

3 Jack P. Gibbs (compilador), Urban Research Methods, Princeton, N. J. Van Nostrand, 1961, pp. xvi-xviii, y Gino Germani, "Urbanización, secularización y desarrollo económico", Revista Mexicana de Sociología, vol. XXV, núm. 2, 1963, pp. $627-629$.

4 Estas definiciones se encuentran en: Dorselaer y Gregory, op. cit., tomo I, pp. 15-18; L. Wirth, "Urbanism as a Way of Life", en Hatt y Reiss (compiladores), Cities and Society, Nueva York, Free Press of Glencoe, 1957, pp. 46-63; P. Hatt y A. Reiss, "The Nature of the City", en Hatt y Reiss, op. cit., p. 17; y otros autores.

5 Hatt y Reiss, loc. cit., p. 18. Véase también Robert Redfield, "The Folk Society", American Journal of Sociology, vol. 52, enero de 1947, pp. 293-308.

6 L. Wirth, loc. cit., p. 50. 
de las conclusiones más importantes alcanzadas por Lewis y Hauser en sus estudios sobre la dicotomía urbano-rural, ${ }^{7}$ la cual puede sintetizarse en la siguiente frase: "se tiene evidencia, todavía no concluyente, de que ambas partes de esta dicotomía, la rural-urbana, representan variables compuestas $\mathrm{y}$, de hecho, sistemas complejos de variables que aún deben ser separadas. Las dicotomías representan probablemente esfuerzos muy apresurados para sintetizar e integrar los pocos conocimientos que se han adquirido a través de la investigación empírica..." 8

Enfoque del continuum urbano-rural. Los estudios mencionados ${ }^{9}$ han destacado de nueva cuenta las deficiencias de la dicotomía urbanorural y han dado lugar al enfoque del continuum urbano-rural, según el cual a medida que se avanza del extremo rural al urbano las localidades manifiestan variaciones regulares en sus patrones de comportamiento. ${ }^{10}$ De acuerdo con este prototipo, la metrópoli de varios millones de habitantes estará más cerca del polo urbano del continuum y la localidad de 100 habitantes del polo opuesto, y se supone que las localidades intermedias se distribuyen a lo largo de la escala del tamaño de las localidades, pudiéndose ubicar empíricamente en determinado punto del continuum. ${ }^{11}$

Enfoque del conjunto de características. Este enfoque conduce a una distinción de índole dicotómica y se diferencia del citado en primer lugar en que los atributos de cada categoría son variables empíricas que se suponen relacionadas causalmente, o bien son causa de otros rasgos de esa misma categoría de población. Así, por ejemplo, Sorokin y Zimmerman ${ }^{12}$ han considerado a la ocupación como la variable que establece cambios cualitativos entre ambos tipos de comunidades. Así, la ocupación agrícola caracteriza a la comunidad rural, y la ocupación no agrícola a la urbana. Junto con la ocupación, la magnitud de la población de la localidad y la densidad de población han sido las variables "causales" más aplicadas en este enfoque, las cuales se supone que guardan relación directa con ciertos patrones socioculturales. ${ }^{13}$

7 Oscar Lewis, "Further Observations on the Folk-Urban Continuum and Urbanization with Special Reference to Mexico City", en P. Hauser y L. Schnore (compiladores), The Study of Urbanization, Nueva York, John Wiley, 1966, pp. 491503 ; y Pinilip Hauser, "Observations on the Urban-Folk and Urban-Rural Dichotomies as Forms of Western Ethnocentrism", ibid., pp. 503-518.

8 Hauser, loc. cit., p. 514.

9 Además de las citadas, se mencionan otras críticas. Ibid., p. 504.

10 Otis Duncan, "Community Size and the Rural-Urban Continuum", en Hatt y Reiss, op. cit., p. 45. Para más detalles ver Stuart Queen y David Carpenter, The American City, Nueva York, McGraw Hill, 1953, p. 38.

11 Entre los ensayos que se han hecho para verificar empíricamente la existencia de un continuum urbano-rural, destaca el elaborado por Duncan con relación a las localidades de los Estados Unidos en 1950, y en el cual se concluye que, a pesar de no existir un continuum matemático, el mencionado enfoque es un instrumento útil para el análisis de las diferencias urbano-rurales. Duncan, op. cit., p. 45.

12 P. Sorokin y C. Zimmerman, Principles of Rural-Urban Sociology, Nueva York, Henry Holt and Co., 1929, citado en Hatt y Reiss, op. cit., p. 18.

13 Gibbs, op. cit., p. 464, y Duncan, op. cit., p. 36. 
Criterios censales $Y$ No censales Para definir la población uRbana

Los criterios censales que se aplican para definir la población urbana pueden agruparse en dos grandes categorías: a) según alguna característica de las menores divisiones administrativas de un país, y $b$ ) según el número de habitantes de las localidades.

Un estudio de las Naciones Unidas sobre las características de los censos de 53 países ${ }^{14}$ da a conocer los países que aplican uno $u$ otro de los criterios mencionados así como las variantes principales. Los resultados del estudio permiten distinguir cerca de 30 distintos tipos de localidades urbanas.

Ante esta diversidad de criterios para definir población urbana, las instituciones internacionales como la Sociedad de Naciones ${ }^{\mathbf{1 5}}$ y hoy día Naciones Unidas, ${ }^{16}$ el Instituto Interamericano de Estadísti$\mathrm{ca}^{17} \mathrm{y}$ otros han hecho esfuerzos por unificar los criterios censales. En virtud de la actual imposibilidad de lograr este propósito, se ha recomendado que además de la definición censal de población urbana y rural de cada país, que establece una dicotomía categórica de la población, se proporcione información para la clasificación de localidades en una serie de grupos de tamaños distintos que representen inflexiones a lo largo del continuum.

Ante esta situación, Naciones Unidas ha optado por utilizar en los estudios comparativos internacionales sobre el proceso de urbanización en América Latina, Africa y Asia un criterio dicotómico según el cual se consideran urbanas las localidades de 20000 y más habitantes. Este límite inferior ha sido establecido lo suficientemente elevado de modo que incluya a la gran mayoría de las localidades que se definen como urbanas según otros criterios. ${ }^{18}$

14 Dorselaer y Gregory, op. cit., pp. 13-14, y Naciones Unidas, "On the Distinction Between Urban and Rural-National Practices and Recommendations", en Gibbs, op. cit., pp. 481-489.

15 Con objeto de llegar a una clasificación urbana-rural tipo, la Sociedad de Naciones propuso en 1938 como criterio básico la proporción de población dedicada a la agricultura (población activa y dependiente), según la cual debieran distinguirse tres grupos: comunas rurales (con $60 \%$ y más), comunas mixtas (con 40 a $60 \%$ ) y comunas urbanas (con menos del $40 \%$ ). Naciones Unidas, loc. cit., pp. $475-477$.

16 Lo infructuoso de los esfuerzos realizados por Naciones Unidas por unificar los criterios censales para definir población urbana se manifiesta en la recomendación con relación al censo de 1970, que dice: "en vista de las diferencias nacionales en las características que distinguen las áreas urbanas de las rurales, la distinción entre la población urbana y la rural no es todavía factible de englobarse en una sola definición que pueda ser aplicable a todos los países..." Naciones Unidas, Principles and Recommendations for the 1970 Population Censuses, Statistical Papers, Serie M, Núm. 44, 1967, p. 63.

17 El Instituto Interamericano de Estadística ha reunido las diversas posibles características que integran una definición de "población urbana", las cuales se exponen detalladamente en su publicación Censo de población-estudios sobre métodos y procedimientos, Washington, Unión Panamericana, 1960.

18 John Durand y César Peláez. "Características de la urbanización en América Latina", en Clyde Kiser (compilador), Componentes de los cambios demográficos en América Latina, Fundación Milbank Memorial, vol. XLIII, núm. 4, octubre de 1965, 2a. parte, pp. 148-149. Con relación al grado de comparabilidad internacional de distintos tipos de información censal sobre localidades, Gibbs y Davis recomiendan utilizar como límite inferior de la clase urbana una población que 
En el caso de México, el censo de población, de acuerdo con el segundo criterio antes citado, considera "urbana" a la población que habita localidades con 2500 o más habitantes y como "rural" la que habita localidades menores. ${ }^{19}$

Con respecto a la aplicabilidad de la anterior definición censal, puede decirse que existe opinión generalizada en cuanto a que el límite de 2500 habitantes no diferencia en forma adecuada la población predominantemente urbana de la rural ${ }^{20}$-lo cual queda de manifiesto en el análisis que se presenta en la segunda parte de este trabajo. Hay un número creciente de estudios sobre México en que se aplican como límites inferiores para definir población urbana los de 5000 , 10000 o aun 20000 habitantes; ${ }^{21}$ en otras investigaciones, en que es estrictamente indispensable utilizar la información estadística censal sobre población urbana y rural, se conserva el límite de 2500 .

\section{UNA NUEVA CLASIFICACIÓN DE POBLACIÓN URBANA Y RURAL}

Con base en los antecedentes presentados, se plantean los requisitos que debe satisfacer una nueva clasificación de población urbana y rural en México:

a) componerse de más de dos grupos de localidades (no dicotómica), por considerarse que una sociedad compuesta de un sector totalmente tradicionalista y otro completamente moderno tiene escasa correspondencia con la realidad del país;

b) fundamentarse en un análisis en dos dimensiones: el tamaño de la población de las localidades, considerada como

no sea menor de 10000 habitantes; aplicar límites entre 2000 y 10000 habitantes introduciría una distorsión en las comparaciones. J. Gibbs y K. Davis, "Conventional versus Metropolitan Data in the International Study of Urbanization", en Gibbs, op. cit., p. 435.

19 El concepto de población urbana se ha captado censalmente a partir de 1910, cuando se consideró como tal la que vivía en localidades de más de 4000 habitantes. En 1921, el límite se redujo a 2000 o más habitantes y a partir de 1930 está vigente el límite de 2500 habitantes. Susana Lerner, Análisis comparativo de los conceptos utilizados en los censos de población, 1895-1960, Centro de Estudios Económicos y Demográficos, El Colegio de México, 1966, mimeografiado.

20 Entre los estudios que han señalado o probado tales deficiencias están: Nathan Whetten, Rural Mexico, Chicago, University of Chicago Press, 1948; Robert Burnright, Nathan Whetten y Bruce Waxman, "La fertilidad diferencial ruralurbana en México", Ciencias y Políticas y Sociales, año IV, núms. 11 y 12, enerojunio 1958; Claudio Stern, Las regiones de México y sus niveles de desarrollo socioeconómico, tesis profesional, Escuela Nacional de Ciencias Políticas y Sociales, UNAM, 1966, Ma Teresa Gutiérrez de MacGregor, Desarrollo y distribución de la población urbana en México, Instituto de Geografía, UNAM, 1965; Floyd Dotson y Lilian Ota Dotson, "Urban Centralization and Decentralization in Mexico", Rural Sociology, núm. 21, marzo de 1956, pp. 41-49; Calixto Rangel Contla, El desarrollo diferencial de México (1940-1960), tesis profesional, Escuela Nacional de Ciencias Políticas y Sociales, UNAM, 1965, y Raúl Benítez Zenteno, "La población urbana y rural de México", Revista Mexicana de Sociología, vol. XXIV, núm. 3, 1962, pp. 689-703.

21 El límite de población urbana de 5000 habitantes se aplicó en los estudios antes citados de Burnright, Whetten y Waxman, y Stern; el límite de 10000 habitantes en los trabajos de Whetten, Gutiérrez MacGregor y Dotson; Rangel Contla utilizó el de 20000 habitantes. 
variable independiente, y cada una de las características socioeconómicas seleccionadas de la población, como variable dependiente; 22

c) expresarse, por razones de facilidad en el manejo estadístico, en función del tamaño de la población de las localidades.

Variables seleccionadas. Las variables dependientes que se enumeran a continuación se consideran representativas de importantes patrones de comportamiento de índole económica social y cultural de la población. ${ }^{23}$

a) proporción de la población económicamente activa dedicada a actividades no agrícolas (PEA no agrícola); ${ }^{24}$

b) proporción de población alfabeta;

c) proporción de la población que ha terminado sus estudios primarios ;

d) proporción de la población que habla español, usa zapatos y vestidos no indígenas (1940), o bien que usa zapatos $(1960) ; 25$

e) proporción de la población asalariada. ${ }^{26}$

22 Se estableció como variable independiente la población de las localidades, porque se supone que las características socioeconómicas y culturales de las mismas, analizadas a través de variables seleccionadas, para distinguir la población urbana de la no urbana, presentan diferencias cuantitativas al variar el tamaño demográfico de las localidades. O sea que en función de la población de las localidades se pretende establecer una clasificación urbana-rural con características de continuum.

23 En apoyo de las consideraciones hechas para la selección de las variables dependientes, excepto la $d$ ), se tomó en cuenta el que en uno de los estudios más recientes sobre la urbanización en México, las variables $a$ ), b), c) y e) ocuparon los rangos primero, segundo, tercero y cuarto, respectivamente, en el índice de urbanización obtenido mediante una matriz de componentes principales de 13 variables. Esto se consideró indicativo de una alta asociación de tales factores con la forma de vida urbana, tal como se definió en el mencionado estudio. Ver Ricardo Cinta, Aspectos socioeconómicos de la urbanización en México, tesis profesional, Escuela Nacional de Ciencias Políticas y Sociales, UNAM, México, 1967, pp. 220, 223 y 228 . Además de las variables seleccionadas se han estudiado otras para el mismo fin, tales como el tamaño de la familia, el índice de masculinidad, la participación femenina en la fuerza de trabajo, el ingreso per capita, servicios públicos $\mathrm{y}$ otras. El número de variables estudiadas se limita en función de la disponibilidad de información y de tiempo para su análisis. El estudio de otras variables puede verse en 0 . Duncan y A. Reiss, Jr., Social Characteristics of Urban and Rural Communities, 1950, Nueva York, John Wiley and Sons, 1956.

${ }^{24} \mathrm{La}$ PEA no agrícola constituye un indicador básico para distinguir la actividad urbana de la no urbana (véase página 3), puesto que, desde el punto de vista ocupacional, la ciudad puede considerarse como el lugar de residencia y de trabajo de grandes grupos de población dedicados predominantemente a actividares secundarias y terciarias. Por esta razón, esta variable es la que se aplica básicamente en los métodos empleados para delimitar áreas urbanas y zonas metropolitanas, tanto en países desarrollados como en países en desarrollo; difiere únicamente el criterio cuantitativo. Ver J. Gibbs, op. cit., pp. 31-77, así como los estudios que se citan en este trabajo de Maçura, Zimmerman, y Sorokin y Davis.

25 Esta variable social, pero con algunos aspectos económicos en lo que se refiere a ingresos, fue la empleada por Whetten en la obtención de un índice que expresara la proporción de población que vive de acuerdo con un patrón cultural, el que denominó indígena colonial y al cual dio mucha importancia en el estudio para caracterizar a la población rural en México. Whetten, op. cit., pp. 260-366.

26 Esta variable, compuesta de obreros, empleados y jornaleros asalariados, 


\section{Cuadro 1}

MÉXICO: NÚMERo de LOCALIdADES SELECCIONADAS : PARA ESTUdIO, POR INTERVALOS DE POBLACIÓN Y POR ENTIDAD FEDERATIVA, 1940

\begin{tabular}{|c|c|c|c|c|c|c|c|c|c|c|}
\hline \multirow{4}{*}{ Entidades } & \multicolumn{10}{|c|}{ localidades por intervalos de poblacion } \\
\hline & \multicolumn{2}{|c|}{$A$} & \multicolumn{2}{|c|}{ 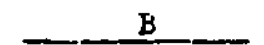 } & \multicolumn{2}{|c|}{$\mathrm{C}$} & \multicolumn{2}{|c|}{ D } & \multicolumn{2}{|c|}{$\mathbf{E}$} \\
\hline & $2.5=$ & 5000 & $5=$ & $10 \overline{000}$ & $10-$ & 15000 & $15=$ & $2 \overline{000}$ & 20000 & $0+$ \\
\hline & $\begin{array}{l}\text { Estú } \\
\text { dia- } \\
\text { das }\end{array}$ & Total & $\begin{array}{l}\text { Estu } \\
d i n=\bar{a} \\
d e s\end{array}$ & Total & $\begin{array}{l}\text { Estu } \\
\text { diam } \\
\text { das }\end{array}$ & Total & $\begin{array}{l}\text { Estux } \\
\text { dia- } \\
\text { das }\end{array}$ & Total & $\begin{array}{l}\text { Estu } \\
\text { dia- } \\
\text { das }\end{array}$ & Total \\
\hline Aguascalientes & - & 3 & - & - & - & - & - & - & 1 & 1 \\
\hline Eaja California Norte & - & 1 & - & - & - & - & 2 & 2 & - & - \\
\hline Baja Califernia Sur & - & 1 & - & 1 & 1 & 1 & - & - & - & - \\
\hline Campeche & - & 4 & - & $t$ & - & - & - & - & 1 & 1 \\
\hline Coahuila & - & 8 & 1 & 9 & - & - & 3 & 3 & 3 & 3 \\
\hline Colima & - & 2 & - & 1 & - & - & - & - & 1 & $i$ \\
\hline Chiapas & 1 & 8 & 1 & 6 & 1 & 1 & 2 & 2 & - & - \\
\hline Chihuahua & - & 12 & - & 5 & 2 & 2 & - & - & 3 & 3 \\
\hline Distrito Federal & - & 10 & - & 5 & 2 & 2 & - & - & - & 1 \\
\hline Durango & - & 13 & - & 2 & - & - & - & - & 2 & 2 \\
\hline Guana jua to & - & 14 & - & 13 & 5 & 5 & 1 & 1 & 4 & 4 \\
\hline Guerrero & - & 18 & - & 5 & 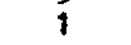 & 1 & - & - & - & - \\
\hline Hidalgo & - & 15 & - & 2 & 2 & 2 & - & - & 1 & 1 \\
\hline Jalisco & - & 34 & - & 21 & 6 & 6 & - & - & 2 & 2 \\
\hline Méxjco & 4 & 48 & - & 11 & - & - & - & - & 1 & $i$ \\
\hline Hichoacán & 1 & 38 & 1 & 14 & 3 & 3 & 1 & 1 & 2 & 2 \\
\hline Morelos & - & 9 & - & 1 & 1 & 1 & - & - & - & - \\
\hline Neyarit & - & 5 & - & 3 & 1 & 1 & 1 & 1 & - & - \\
\hline Muevc leon & 1 & 8 & - & 4 & - & - & - & - & 1 & 1 \\
\hline Caxaca & 14 & 32 & 3 & 4 & 1 & 1 & - & - & 1 & 1 \\
\hline Puebla & 5 & 36 & 1 & 9 & $\sim$ & - & 2 & 2 & 1 & 1 \\
\hline Querétaro & - & 2 & - & 1 & - & - & - & - & 1 & 1 \\
\hline Quintana Roo & - & 1 & - & - & $\sim$ & - & - & - & - & - \\
\hline San Luis Potcs 1 & - & 11 & - & 6 & - & - & 1 & 1 & 1 & 1 \\
\hline Sinaloa & - & 5 & - & 3 & 1 & 1 & - & - & 2 & 2 \\
\hline Sonora & - & 11 & - & 2 & 4 & 4 & 1 & 1 & - & - \\
\hline Tabasco & - & 6 & - & 1 & - & - & - & - & 1 & 1 \\
\hline Tamaul ipas & - & 1 & - & 4 & - & - & 2 & 2 & 3 & 3 \\
\hline Tlaxcala & 4 & 11 & 1 & 4 & - & - & - & - & - & - \\
\hline Veracru & 3 & 38 & 1 & 10 & 5 & 5 & 2 & 2 & 3 & 3 \\
\hline Yucatán & 5 & 13 & 2 & 8 & 1 & 1 & - & - & 1 & 1 \\
\hline Zacatecas & 1 & 18 & - & 4 & - & - & - & - & 2 & 2 \\
\hline Total & 39 & 436 & 12 & 160 & 37 & 37 & 18 & 18 & $38^{\circ}$ & 39 \\
\hline
\end{tabular}

Fuente: VI Censo General de Población.

a Municipios cuya localidad principal tenía $80 \%$ y más de la población municipal.

b No se incluyó la ciudad de México.

Se supone que estas variables son lo bastante sensibles para captar diferencias a distintos tamaños de localidades, esperándose encontrar, predominantemente, valores bajos de las variables en las localidades de menor población y un incremento en tales valores a medida que aumente el tamaño demográfico de las localidades.

Datos. En México no se cuenta con estadísticas censales al nivel de localidad, exceptuado el caso de las localidades de 10000 y más se considera un indicador de "burocratización" de la fuerza de trabajo, índice concomitante de la división de trabajo $\mathrm{y}$, por lo tanto, característica básica de lo urbano. Véase W. Moore, Social Change, Englewood Cliffs, N. J., Prentice-Hall, 1963, citado por R. Cinta, op. cit., p. 129. 
Cuadro 2

México: NÚMERo de localidades SEIECCIONADAS PARA ESTUdio, POR INTERVALOS DE POBLACTON Y POR ENTIDAD FEDERATTVA, 1960

\begin{tabular}{|c|c|c|c|c|c|c|c|c|c|c|}
\hline \multirow[b]{2}{*}{ Entidades } & \multicolumn{10}{|c|}{ Locelldedes por intervalos de pobleoibn } \\
\hline & $\frac{2.5-}{\text { ditu }}$ & $\frac{5000}{\operatorname{sotin1}}$ & $\begin{array}{l}\frac{5}{\text { Hetu }} \\
\text { disay }\end{array}$ & $\frac{\mathrm{B}}{10000}$ & $\begin{array}{l}\frac{10=}{\text { jetu }} \\
\text { dia- } \\
\text { dast }\end{array}$ & $\frac{\frac{c}{15000}}{\text { Total }}$ & $\frac{15=}{\text { Estu }}$ & $\frac{\mathrm{D}}{20000}$ & 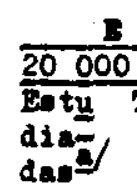 & $\frac{0+}{\text { Total }}$ \\
\hline 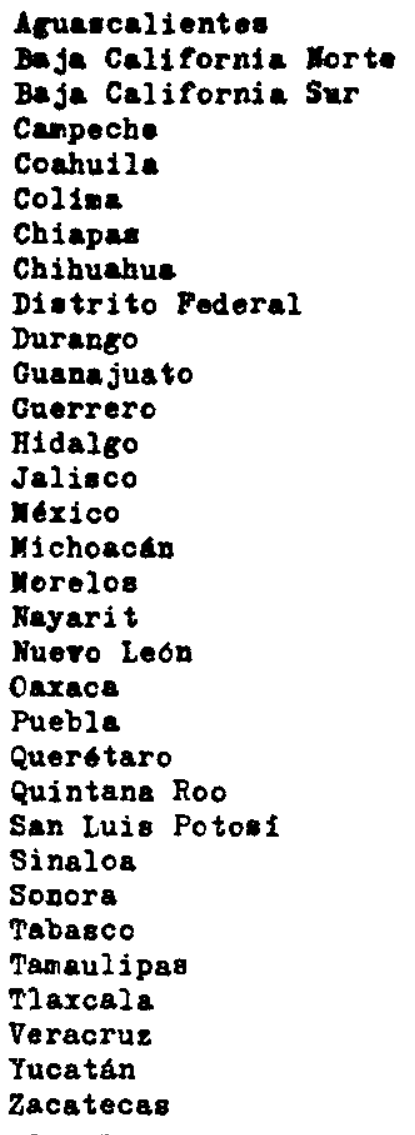 & $\begin{array}{l}- \\
\overline{-} \\
\overline{1} \\
- \\
- \\
- \\
- \\
- \\
- \\
- \\
- \\
3 \\
- \\
- \\
- \\
13 \\
8 \\
- \\
- \\
- \\
- \\
2 \\
- \\
1 \\
5 \\
3 \\
-\end{array}$ & $\begin{array}{r}2 \\
5 \\
0 \\
10 \\
11 \\
4 \\
25 \\
17 \\
19 \\
20 \\
26 \\
38 \\
17 \\
52 \\
95 \\
59 \\
21 \\
13 \\
6 \\
54 \\
69 \\
5 \\
1 \\
17 \\
10 \\
14 \\
12 \\
14 \\
17 \\
56 \\
24 \\
14\end{array}$ & $\begin{array}{l}0 \\
- \\
- \\
1 \\
-1 \\
- \\
- \\
- \\
- \\
- \\
- \\
1 \\
1 \\
- \\
- \\
- \\
- \\
5 \\
3 \\
- \\
- \\
- \\
- \\
- \\
- \\
-1 \\
- \\
2 \\
- \\
-\end{array}$ & $\begin{array}{r}2 \\
3 \\
1 \\
1 \\
6 \\
0 \\
5 \\
7 \\
7 \\
5 \\
10 \\
10 \\
9 \\
23 \\
21 \\
24 \\
4 \\
4 \\
3 \\
16 \\
17 \\
1 \\
0 \\
6 \\
8 \\
6 \\
5 \\
3 \\
5 \\
36 \\
9 \\
13\end{array}$ & $\begin{array}{l}- \\
- \\
- \\
- \\
1 \\
- \\
- \\
1 \\
- \\
- \\
- \\
-1 \\
- \\
- \\
2 \\
1 \\
- \\
- \\
3 \\
- \\
- \\
- \\
- \\
- \\
- \\
- \\
-\end{array}$ & 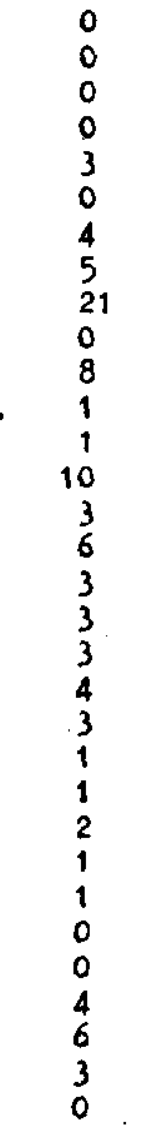 & $\begin{array}{l}- \\
- \\
- \\
- \\
- \\
1 \\
- \\
- \\
- \\
- \\
- \\
- \\
- \\
- \\
- \\
- \\
- \\
- \\
- \\
- \\
- \\
-3 \\
- \\
- \\
- \\
-\end{array}$ & $\begin{array}{l}0 \\
0 \\
0 \\
0 \\
2 \\
2 \\
1 \\
2 \\
10 \\
1 \\
2 \\
1 \\
0 \\
6 \\
0 \\
2 \\
0 \\
0 \\
1 \\
1 \\
2 \\
0 \\
0 \\
1 \\
1 \\
3 \\
0 \\
2 \\
1 \\
6 \\
1 \\
1\end{array}$ & $\begin{array}{l}1 \\
1 \\
1 \\
1 \\
5 \\
1 \\
2 \\
3 \\
- \\
- \\
1 \\
-1 \\
1 \\
2 \\
-1 \\
- \\
-1 \\
1 \\
1 \\
1 \\
- \\
-1 \\
1 \\
2 \\
2 \\
-3 \\
3 \\
-3 \\
1 \\
1 \\
1\end{array}$ & $\begin{array}{l}1 \\
3 \\
1 \\
2 \\
7 \\
1 \\
3 \\
4 \\
20 \\
2 \\
9 \\
2 \\
2 \\
5 \\
2 \\
7 \\
1 \\
1 \\
2 \\
1 \\
3 \\
1 \\
-2 \\
2 \\
3 \\
6 \\
1 \\
7 \\
- \\
8 \\
1 \\
2\end{array}$ \\
\hline Total & 44 & 747 & 18 & 272 & 12 & 97 & 6 & 49 & 34 & $110 \mathrm{~b}$ \\
\hline
\end{tabular}

Fuente: VIII Censo General de Población.

a Municipios cuya localidad principal tenía $80 \%$ y más de la población municipal.

b Estas corresponden a las localidades según el censo; en caso de considerarse "áreas urbanas" (la ciudad central y su periferia de características urbanas), su rúmero se reduciría a 92, pues 18 de las localidades del Distrito Federal están integradas en su área urbana.

habitantes en 1940 , sobre las cuales el censo de población proporciona la misma información que al nivel estatal. Por lo tanto, a efecto de completar la selección de localidades para el estudio, se escogieron todas las localidades de 2500 y más habitantes cuya población fuera como mínimo el $80 \%$ de la población de su municipio. En este caso se supuso que el dato censal del municipio representa el de la localidad y es susceptible de estudiarse como tal.

Así, en 1940, la selección de localidades mayores de 2500 habitantes se integró con 144 (de las cuales 93 eran mayores de 10000 habi- 
tantes) y en 1960 con 114 centros de población (véanse los cuadros 1 y 2). En los 165 casos en que se utilizaron datos municipales, el promedio de población de la localidad principal con respecto a la de su municipio fue del $90 \%$; en varios de estos municipios su población coincidió con la de la localidad escogida.

Las condiciones impuestas para la selección de las localidades dio por resultado que tal selección manifestara diferencias estructurales

\section{Cuadro 3}

MÉxico: NúMERo (N) Y PORCIENTO ACUMULAdo (\%) DE LOCALIDADES, SEGÚN DIFERENTES CARACTERÍSTICAS, 1940

3.1 Según proporción de población económlcamente activa no agrícola

\begin{tabular}{|c|c|c|c|c|c|c|c|c|c|c|}
\hline \multirow{4}{*}{$\begin{array}{l}\text { Interva- } \\
\text { los por- } \\
\text { centua- } \\
\text { les }\end{array}$} & \multicolumn{10}{|c|}{ Localidades por intervalos de población (habitantes) } \\
\hline & \multicolumn{2}{|r|}{ A } & \multicolumn{2}{|c|}{$\mathrm{B}$} & \multicolumn{2}{|c|}{$\mathrm{C}$} & \multicolumn{2}{|c|}{$\mathrm{D}$} & \multicolumn{2}{|c|}{$\mathbf{E}$} \\
\hline & \multicolumn{2}{|c|}{$2.5-5,000$} & \multicolumn{2}{|c|}{$5-10,000$} & \multicolumn{2}{|c|}{$20-15,000$} & \multicolumn{2}{|c|}{$15-20,000$} & \multirow{2}{*}{$\frac{20,000}{N}$} & \multirow{2}{*}{$\frac{+}{q}$} \\
\hline & $\bar{N}$ & $\%$ & $\mathrm{~N}$ & $\%$ & $\mathbf{K}$ & $\phi$ & $\mathbf{N}$ & $\%$ & & \\
\hline $0-10$ & 16 & (4I) & 0 & - & 0 & - & 0 & - & 0 & - \\
\hline $10-20$ & 10 & (67) & 3 & (25) & 0 & - & 0 & - & 0 & - \\
\hline $20-30$ & 5 & (79) & 4 & $(58)$ & 0 & - & 0 & - & 0 & - \\
\hline $30-40$ & 3 & $(87)$ & 2 & (75) & 3 & $(8)$ & 0 & - & 0 & - \\
\hline $40-50$ & 2 & (92) & 1 & $(83)$ & 3 & $(16)$ & 0 & - & 0 & - \\
\hline $50-60$ & 1 & (95) & 0 & $(83)$ & 4 & (27) & 1 & $(6)$ & 1 & (3) \\
\hline $60-70$ & 1 & (97) & 1 & (92) & 5 & (47) & 0 & $(6)$ & 0 & (3) \\
\hline $70-80$ & 0 & $(97)$ & 0 & $(92)$ & 5 & $(54)$ & 7 & $(44)$ & 6 & (28) \\
\hline $80-90$ & 1 & $(100)$ & 1 & $(100)$ & 11 & $(84)$ & 7 & $(83)$ & 12 & $(50)$ \\
\hline $90-100$ & 0 & - & 0 & - & 6 & $(100)$ & 3 & $(100)$ & 19 & $(100)$ \\
\hline Sumas & 39 & & 12 & & 37 & & 18 & & 38 & \\
\hline
\end{tabular}

3.2 Según proporción de población de 6 años y más que sab leer y escribir

\begin{tabular}{rrrrr|rrrrrrr}
\hline $0-10$ & 11 & $(28)$ & 1 & $(8)$ & 0 & - & 0 & - & 0 & - \\
$10-20$ & 7 & $(46)$ & 2 & $(25)$ & 0 & - & 0 & - & 0 & - \\
$20-30$ & 7 & $(64)$ & 0 & $(25)$ & 1 & $(3)$ & 0 & - & 0 & - \\
$30-40$ & 5 & $(77)$ & 3 & $(50)$ & 1 & $(5)$ & 0 & - & 0 & - \\
$40-50$ & 5 & $(90)$ & 2 & $(67)$ & 5 & $(19)$ & 0 & - & 0 & - \\
$50-60$ & 3 & $(97)$ & 1 & $(75)$ & 14 & $(57)$ & 2 & $(11)$ & 7 & $(18)$ \\
$60-70$ & 1 & $(100)$ & 2 & $(92)$ & 7 & $(76)$ & 8 & $(56)$ & 8 & $(39)$ \\
$70-80$ & 0 & $(100)$ & 1 & $(100)$ & 7 & $(95)$ & 3 & $(72)$ & 18 & $(87)$ \\
$80-90$ & 0 & - & 0 & - & 2 & $(100)$ & 5 & $(100)$ & 5 & $(100)$ \\
$90-100$ & 0 & - & 0 & - & 0 & - & 0 & - & 0 & - \\
Sumes & 39 & & 12 & & 37 & & 18 & & 38 &
\end{tabular}




\section{Cuadro 3 (continuación)}

3.3 segín proporción de poblectón de 6 afos y wís que ha rectbido 6 años de instrucción primerla

\begin{tabular}{|c|c|c|c|c|c|c|c|c|c|c|}
\hline \multirow{3}{*}{$\begin{array}{l}\text { Interva- } \\
\text { los por- } \\
\text { centwa } \\
\text { les } 8\end{array}$} & \multicolumn{10}{|c|}{ Localidades por intervalos de población (habitantes) } \\
\hline & \multicolumn{2}{|r|}{$\mathbf{A}$} & \multicolumn{2}{|c|}{ B } & \multicolumn{2}{|c|}{ c. } & \multicolumn{2}{|r|}{$\mathbf{D}$} & \multicolumn{2}{|l|}{$\mathbf{E}$} \\
\hline & 2.5 & 5,000 & $5-$ & 10,000 & 10 & 15,000 & 15 & 20,000 & 20,000 & + \\
\hline a. 3.5 & 36 & (92) & 7 & (58) & 1 & (3) & $\mathbf{0}$ & - & $\mathbf{0}$ & - \\
\hline $3.5-7.0$ & 1 & (95) & 3 & (83) & 6 & (19) & 0 & - & 0 & - \\
\hline 7.0 .10 .5 & 0. & (95) & 1 & (92) & 14 & (57) & 2 & (11) & 3 & (8) \\
\hline $10.5-14.0$ & 1 & (97) & 0 & (92) & 8 & $(78)$ & 5 & (29) & 6 & (24) \\
\hline $14.0-17.5$ & 1 & $(100)$ & 1 & $(100)$ & 4 & (89) & 4 & (61) & 7 & (42) \\
\hline $17.5-21.0$ & 0 & - & 0 & - & 1 & (92) & 2 & (T2) & 8 & (63) \\
\hline $22.0-24.5$ & 0 & - & 0 & - & 2 & (97) & 2 & (83) & 5 & (76) \\
\hline $24.5-28.0$ & 0 & - & 0 & - & 1 & $(100)$ & 3 & $(100)$ & 5 & (89) \\
\hline $28.0-31.5$ & 0 & - & 0 & - & 0 & $=$ & 0 & - & 2 & (95) \\
\hline $31.5-35.0$ & 0 & - & 0 & - & 0 & - & 0 & - & 2 & $(100)$ \\
\hline Stras & 39 & & 12 & & 37 & & 18 & & 38 & \\
\hline
\end{tabular}

3.4 Segin proporetón de la población que habla lengua española o.-1d1oman extranjero y usa zapatos e indwentarla de tipo no Indígena

\begin{tabular}{rrrrr|rrrrrrr}
\hline $0-10$ & 28 & $(72)$ & 5 & $(42)$ & 1 & $(3)$ & 0 & - & 0 & - \\
$10-20$ & 3 & $(79)$ & 3 & $(67)$ & 0 & $(3)$ & 0 & - & 0 & - \\
$20-30$ & 2 & $(85)$ & 0 & $(67)$ & 0 & $(3)$ & 0 & - & 0 & - \\
$30-40$ & 3 & $(92)$ & 1 & $(75)$ & 2 & $(8)$ & 0 & - & 0 & - \\
$40-50$ & 0 & $(92)$ & 0 & $(75)$ & 0 & $(8)$ & 0 & - & 0 & - \\
$50-60$ & 1 & $(95)$ & 0 & $(75)$ & 1 & $(11)$ & 1 & $(6)$ & 0 & - \\
$60-70$ & 0 & $(95)$ & 0 & $(75)$ & 5 & $(24)$ & 0 & $(6)$ & 3 & $(8)$ \\
$70-80$ & 0 & $(95)$ & 0 & $(75)$ & 8 & $(46)$ & 4 & $(28)$ & 4 & $(18)$ \\
$80-90$ & 1 & $(97)$ & 1 & $(83)$ & 9 & $(70)$ & 4 & $(50)$ & 6 & $(34)$ \\
$0-100$ & 1 & $(100)$ & 2 & $(100)$ & 11 & $(100)$ & 9 & $(100)$ & 25 & $(100)$ \\
$8=000$ & 39 & & 12 & & 37 & & 18 & & 38 & \\
\hline
\end{tabular}

Fuente: VI Censo General de Población.

Nota: En los intervalos A y B las localidades se componen de municipios cuya localidad principal tiene $80 \%$ más de la población municipal.

a En el cuadro 3.3, el valor máximo de esta variable fue próximo a $35.0 \%$, por lo que se tomó como límite superior este nivel y se dividió en deciles, a efecto de lograr mayor sensibilidad en el comportamiento de la variable en los distintos intervalos de población.

en los intervalos que la componen, por lo que el análisis comparativo que se hace de las clasificaciones obtenidas para 1940 y 1960 debe tomarse con las reservas del caso.

Clasificación urbano-rural tipo continuum. A efecto de construir una clasificación de población según el tamaño de las localidades, como representación operativa de un continuum rural-urbano, se ana- 
lizan las relaciones entre la población de las localidades y ciertas características socioeconómicas de las mismas que se consideran asociadas con la forma de vida urbana.' De este modo y de acuerdo con los lineamientos generales del enfoque del "conjunto de características" antes expuesto, se supone que el carácter urbano (o rural) de una localidad está dado en función del tipo de actividad económica desarrollada y del nivel sociocultural alcanzado. Siguiendo este razonamiento, serán más urbanas (o menos rurales) aquellas localidades que

\section{Cuadro 4}

MÉXico: NúMero (N) Y PORCiENTo acumulado (\%) DE LOCAlidades, SEGÚN DIFERENTES CARACTERÍSTICAS, 1960

\subsection{Segín proporción de población económicamonte activa no agricola}

\begin{tabular}{|c|c|c|c|c|c|c|c|c|c|c|}
\hline \multirow{4}{*}{$\begin{array}{l}\text { Interva- } \\
\text { los por- } \\
\text { centua- } \\
\text { les }\end{array}$} & \multicolumn{10}{|c|}{ Iocalldades por intervalos de pobleción (hebitantes) } \\
\hline & \multirow{3}{*}{\multicolumn{2}{|c|}{$\frac{A}{\frac{2.5-5,000}{4}}$}} & \multirow{3}{*}{\multicolumn{2}{|c|}{$\frac{B}{\frac{5-10,000}{8}}$}} & \multirow{2}{*}{\multicolumn{2}{|c|}{$\frac{C}{10-15,000}$}} & \multirow{2}{*}{\multicolumn{2}{|c|}{$\frac{D}{15-20,000}$}} & \multicolumn{2}{|c|}{$\mathrm{E}$} \\
\hline & & & & & & & & & \multirow{2}{*}{\multicolumn{2}{|c|}{$\frac{20,000+}{1}$}} \\
\hline & & & & & 13 & 4 & 1 & 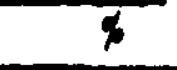 & & \\
\hline $0-10$ & 13 & $(30)$ & 3 & (17) & 0 & - & 0 & - & 0 & - \\
\hline $10-20$ & 10 & (52) & 8 & (6l) & $\mathbf{0}$ & - & 0 & - & 0 & - \\
\hline $20-30$ & 7 & (68) & 0 & (61) & 1 & (8) & 1 & (17) & 0 & - \\
\hline $30-40$ & 5 & $(80)$ & 3 & $(78)$ & 2 & (25) & 0 & (17) & 0 & - \\
\hline 40-50. & 3 & $(86)$ & 0 & $(78)$ & 3 & $(50)$ & 0 & (17) & 1 & (3) \\
\hline $50-60$ & .2 & (91) & 1 & (83) & 1 & (58) & 0 & (27) & 1 & (5) \\
\hline $60-70$ & 2 & (95) & 2 & (94) & 2 & (75) & 3 & $(67)$ & 8 & (29) \\
\hline $70-80$ & 1 & (98) & 1 & $(100)$ & 3 & $(100)$ & 2 & $(100)$ & 9 & (56) \\
\hline $80-90$ & 0 & (98) & 0 & - & 0 & - & 0 & - & 11 & (88) \\
\hline $90-100$ & 1 & $(100)$ & 0 & - & 0 & - & 0 & - & 4 & $(100)$ \\
\hline Sumas & 44 & & 18 & & 12 & & 6 & & 34 & \\
\hline \multicolumn{11}{|c|}{ 4.2 Segín proporción de población de 6 años y más que sabe leer y escribir } \\
\hline $0-10$ & 0 & - & 0 & - & 0 & - & 0 & - & 0 & - \\
\hline $10-20$ & 4 & (9) & 0 & - & 0 & - & 0 & - & 0 & - \\
\hline $20-30$ & 5 & $(20)$ & 2 & (11) & 0 & - & 0 & - & 0 & - \\
\hline $30-40$ & 5 & (32) & 4 & (33) & 0 & $=$ & 0 & - & 0 & - \\
\hline $40-50$ & 13. & (57) & 4 & (56) & 0 & $=$ & 1 & (17) & 0 & - \\
\hline $50-60$ & 8 & (75) & 5. & (83) & 3 & (25) & 0 & (17) & 2 & (6) \\
\hline $60-70$ & 5 & (86) & 0 & (83) & 3 & $(50)$ & $\dot{I}$ & (33) & 5 & (21) \\
\hline $70-80$ & 3 & (93) & 1 & $(89)$ & 3 & (75) & 2 & (67) & 7 & (42) \\
\hline $80-90$ & 3 & $(200)$ & 2 & $(100)$ & 3 & $(100)$ & 2. & $(100)$ & 20 & $(100)$ \\
\hline $90-100$ & 0 & - & 0 & - & 0 & - & 0 & - & 0 & - \\
\hline Sumas & 44 & & 18 & & 12 & & 6 & & 34 & \\
\hline
\end{tabular}




\section{Cuadro 4 (continuación)}

4.3 Según proporclón de poblactón de 6 años y más, con 6 años y más, con 6 años de estudios terminados y aprobados

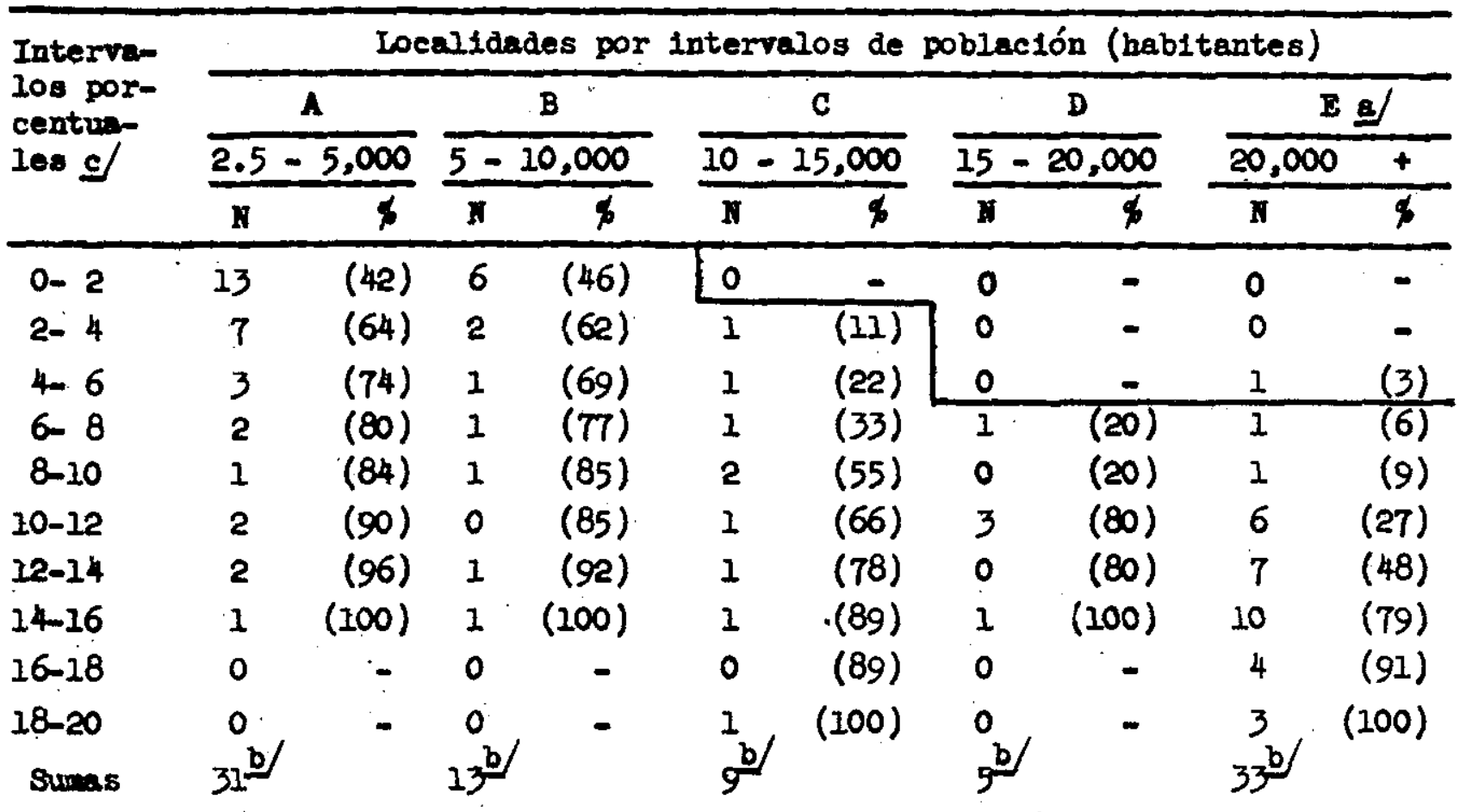

4.4 Según proporción de población que usa zapatos

\begin{tabular}{lrrrr|rrrrrrr}
\hline $0-10$ & 14 & $(32)$ & 4 & $(22)$ & 0 & - & 0 & - & 0 & - \\
$10-20$ & 5 & $(43)$ & 3 & $(39)$ & 0 & - & 0 & - & 0 & - \\
$20-30$ & 6 & $(57)$ & 3 & $(55)$ & 0 & - & 0 & - & 0 & - \\
$30-40$ & 6 & $(70)$ & 0 & $(55)$ & 2 & $(17)$ & 0 & - & 0 & - \\
$40-50$ & 5 & $(82)$ & 3 & $(72)$ & 1 & $(25)$ & 0 & - & 0 & - \\
$50-60$ & 0 & $(82)$ & 1 & $(78)$ & 0 & $(25)$ & 1 & $(17)$ & 3 & $(21)$ \\
$60-70$ & 2 & $(86)$ & 2 & $(89)$ & 3 & $(50)$ & 0 & $(16)$ & 2 & $(36)$ \\
$70-80$ & 4 & $(95)$ & 2 & $(100)$ & 3 & $(75)$ & 1 & $(33)$ & 10 & $(57)$ \\
$80-90$ & 2 & $(100)$ & 0 & - & 3 & $(100)$ & 3 & $(83)$ & 16 & $(100)$ \\
$90-100$ & 0 & - & 0 & - & 0 & - & 1 & $(100)$ & 3 & - \\
5000 & 44 & & 18 & & 12 & & 6 & & 34 &
\end{tabular}

(continúa)

acusen simultáneamente mayor población y más elevados niveles de desarrollo socioeconómico. ${ }^{27}$

El análisis se realizó mediante cuadros de doble entrada en que la. variable independiente - población de las localidades - se registró en el eje horizontal, dividida en varios intervalos, ${ }^{28} \mathrm{y}$ en el eje vertical

27 Esta es la parte principal del procedimiento seguido y está basada en el método aplicado por Maçura para definir la población urbana en la República de Serbia, Yugoslavia. Milos Maçura, "The Influence of the Definition of the Urban Place on the Size of the Urban Population", en J. Gibbs, op. cit., pp. 21-23.

28 Para efectos del análisis la escala de la población de las localidades se limitó y subdividió en puntos específicos seleccionados o determinados ex profeso: 


\section{Cuadro 4 (continuación)}

\subsection{Seeus proporción de asalariados de la población económicanenta activa}

\begin{tabular}{|c|c|c|c|c|c|c|c|c|c|c|}
\hline \multirow{4}{*}{$\begin{array}{l}\text { Interva- } \\
\text { los por- } \\
\text { centus- } \\
\text { les }\end{array}$} & \multicolumn{10}{|c|}{ Localidades por intervalos de población (habitantes) } \\
\hline & \multicolumn{2}{|r|}{ A } & \multicolumn{2}{|c|}{$\mathrm{B}$} & \multicolumn{2}{|c|}{$\mathrm{C}$} & \multicolumn{2}{|c|}{$\mathrm{D}$} & \multicolumn{2}{|c|}{$E^{2}$} \\
\hline & \multicolumn{2}{|c|}{$2.5-5,000$} & \multicolumn{2}{|c|}{$5-10,000$} & \multicolumn{2}{|c|}{$10-15,000$} & \multicolumn{2}{|c|}{$15-20,000$} & \multirow{2}{*}{$\frac{20,000}{N}$} & \multirow{2}{*}{$\frac{+}{\%}$} \\
\hline & $N$ & $1 \%$ & $\mathrm{~N}$ & $\%$ & $\mathbf{N}$ & क & $\mathrm{N}$ & $\phi$ & & \\
\hline $0-10$ & 0 & - & 0 & - & 0 & - & 0 & - & 0 & - \\
\hline $10-20$ & 2 & (5) & 0 & - & 0 & - & 0 & - & 0 & - \\
\hline $20-30$ & 2 & (9) & 2 & (11) & 0 & - & 0 & - & 0 & - \\
\hline $30-40$ & 6 & (23) & 1 & (17) & 0 & - & 0 & - & 0 & - \\
\hline $40-50$ & 7 & (39) & 4 & (39) & 1 & (8) & 0 & - & 0 & - \\
\hline $50-60$ & 7 & (55) & 4 & (61) & 2 & (75) & 0 & - & 1 & (3) \\
\hline $60-70$ & 10 & (77) & 2 & (72) & 1 & (33) & 1 & (17) & 4 & (15) \\
\hline $70-80$ & 3 & (84) & 3 & (89) & 3 & (58) & 2 & (50) & 18 & (68) \\
\hline $80-90$ & 6 & (98) & 2 & $(100)$ & 5 & $(100)$ & 3 & $(100)$ & 11 & $(100)$ \\
\hline $90-100$ & 1 & $(100)$ & 0 & - & 0 & - & 0 & - & 0 & - \\
\hline Sumes & 44 & & 18 & & 12 & & 6 & & 34 & \\
\hline
\end{tabular}

Fuente: VIII Censo General de Población.

Nota: En todos los intervalos las localidades se componen de municipios cuya localidad principal tiene $80 \%$ y más de la población municipal.

a La selección de localidades no incluye a la ciudad de México.

b En el cuadro 4.3 la selección de localidades de estudio se redujo por no contar con los datos correspondientes al estado de Oaxaca.

c En el cuadro 4.3 el valor máximo de esta variable fue próximo a $20 \%$, por lo que se tomó como límite superior este nivel y se dividió en deciles, a efecto de lograr mayor sensibilidaả en el comportamiento de la variable en los distintos intervalos de población.

las variables dependientes, en porcientos expresados en 10 intervalos de clase (véanse los cuadros 3 y 4 ).

Los valores relativos establecidos, de manera arbitraria, para calificar la función ocupacional o el nivel sociocultural de una localidad como predominantemente urbana o rural, fueron, respectivamente, más del $70 \%$ y menos del $30 \%$ de las variables dependientes. ${ }^{29}$

$2500,5000,10000,15000$ y 20000 habitantes. El extremo inferior se fijó en 2500 por ser éste el límite censal actual de población urbano-rural y, básicamente, por considerarse el valor mínimo factible para delimitar la población rural en México. El extremo superior se estableció en los 20000 habitantes, por ser el límite censal y no censal más elevado de cuantos se han empleado a la fecha. Los puntos intermedios de 5000 y 10000 habitantes se establecieron con base en los estudios realizados al respecto por Stern, op. cit., y Whetten, op. cit., respectivamente. La selección de 15000 habitantes es resultado de un estudio preliminar sobre la aplicación del enfoque de Maçura a las localidades de 10000 y más habitantes en 1940.

29 A pesar de lo arbitrario de los valores establecidos, el límite superior fue fijado, en parte, con base en el examen preliminar que se realizó de la PEA no agrícola de las localidades en 1940. En otros métodos aplicados para propósitos análogos al presente, como el de Maçura, se utilizó más de 70 y menos de $40 \%$. Bunle propuso para definir población urbana y rural, más de 60 y menos de $40 \%$, respectivamente ("Rapport de la Commission pour la Definition de la Popu- 


\section{ANÁlisis Y RESULTADOS}

Con objeto de estudiar con mayor facilidad el comportamiento de las variables dependientes a lo largo del tamàno de la población de las localidades e identificar en esta última la formación de uno o varios puntos de inflexión, se empleó como artificio lo que se denominó "la línea del cero porciento". Esta línea, que se señala en los cuadros 3 y 4, une los puntos que en cada intervalo de población tienen $0 \%$ de localidades (o en algunos casos $10 \%$ ) y permite inferir, grosso modo, la existencia de un continuum en las variables estudiadas.

Del examen de la variable PEA no agrícola en 1940 (cuadro 3.1) mediante este procedimiento, se observa una variación escalonada discontinua a medida que aumenta el tamaño de las localidades, hasta alcanzar los 15000 habitantes, punto a partir del cual el cambio se hace poco notorio.

En los intervalos de población $D$ y $E, 30$ la "línea del cero porciento" señala diferencias poco significativas, pues sólo el 6 y el $3 \%$, respectivamente, caen fuera de lo que se considera urbano. Por el contrario, el intervalo $D$ difiere sustancialmente del $C$ por contener este último sólo el $59 \%$ de sus localidades arriba del límite superior establecido. Destaca así el de 15000 habitantes como punto de inflexión a partir del cual se establece una distinción urbana y no urbana. Establecer tal límite en los 20000 habitantes implicaría rechazar un $95 \%$ de las localidades entre 15000 y 20000 habitantes, que alcanzaron una PEA no agrícola, en promedio, del $81 \%$ (17 de 18 localidades).

Mediante un análisis comparativo análogo de los intervalos $A$ y $B$, se concluye que el punto en los 5000 habitantes constituye una división poco clara para distinguir las localidades rurales de las no rurales. ${ }^{31}$

De lo anterior puede inferirse que en la variable PEA no agrícola, las diferencias de la población no son dicotómicas; el límite establecido de lo urbano no coincide con el rural, sino que existe un grupo de localidades entre ambos límites -5000 y 15000 habitantes- que manifiestan características tanto rurales como urbanas. A este grupo de localidades se le denomina localidades mixtas. Dentro de este intervalo de población, destaca el punto de 10000 habitantes como

Iation Rurale", Bulletin de l'Institut International de Statistiques, La Haya, 1938, pp. 158-163). Sobre el límite superior se pueden mencionar los métodos internacionales establecidos para delimitar áreas urbanas y zonas metropolitanas, caso en el cual se emplea más del $65 \%$ de PEA no agrícola. Ver detalles en International Urban Research Institute of International Studies, The World's Metropolitan Areas, Berkeley, 1959, y O. Boustedt, "The Delimitation of Urban Areas", en Gibbs, op. cit., pp. 41-45.

30 Por razones de brevedad, se identifican los intervalos de población de la manera siguiente: $A$, de 2500 a 5000 habitantes; $B$, de 5000 a 10000 habitantes; $C$, de 10000 a 15000 habitantes; $D$, de 15000 a 20000 habitantes, y $E$, de 20000 y más habitantes.

31 Puede conjeturarse que de haberse estudiado un intervalo $A$ con localidades menores de 2500 habitantes, la proporción de localidades con 30 y menos porciento de PEA no agrícola sería mayor $\mathrm{y}$, por lo tanto, más destacada la distinción en los 5000 habitantes. Tanto Stern como Burnight, Whetten y Waxman, manejando algunas variables diferentes, obtuvieron resultados positivos a este respecto (Stern, op. cit., pp. 151-155; Burnright, Whetten y Waxman, loc. cit.). 
inflexión intermedia que claramente distingue las localidades de los intervalos $B$ y $C$. Lo evidencia el hecho de que en el $B$ el $58 \%$ de las localidades son rurales desde un punto de vista ocupacional, mientras que en el $C$ todas las localidades, también con respecto a la ocupación, son de índole no rural. Asimismo, el intervalo $C$ tenía $50 \%$ de sus localidades con atributos predominantemente urbanos; en cambio, el $B$ contaba sólo con el $8 \%$.

Por lo tanto, el grupo mixto de localidades está formado por dos intervalos, $B$ y $C$. El primero inicia la pérdida, desde el punto de vista ocupacional, de características rurales (la proporción de PEA no agrícola nunca es menor del $10 \%$ ), y el segundo registra la obtención marcada de rasgos urbanos (la proporción de PEA no agrícola en todos los casos es mayor del $30 \%$ ). De este modo, a las localidades de 5000 a 10000 habitantes se les puede denominar mixtas rurales, y a las de 10000 a 15000 habitantes mixtas urbanas.

$\mathrm{La}$ aplicación del mismo procedimiento respecto a las tres variables restantes, o sean las $b$ ), c) y $d$ ) (véase p. 6) permite observar en los cuadros 3.2 a 3.4 comportamientos análogos a los de la PEA no agrícola.

La claridad con que se señalan las inflexiones en los puntos de 5000,10000 y 15000 habitantes en variables de la significación del alfabetismo y del idioma y el vestido, especialmente en 1940, refuerzan las inferencias alcanzadas con el estudio de la variable PEA no agrícola, de tal modo que la clasificación de las localidades queda formada en principio de los cuatro grupos siguientes:

1) localidades rurales: menores de 5000 habitantes;

2) localidades mixtas-rurales: entre 5000 y 10000 habitantes;

3) localidades mixtas-urbanas: entre 10000 y 15000 habitantes;

4) localidades urbanas: mayores de 15000 habitantes.

Para confirmar los primeros resultados obtenidos y determinar si la clasificación urbana-rural establecida para 1940 cambia con el tiempo, se elaboró un análisis semejante para 1960 , con todas las variables dependientes enumeradas previamente. Las variaciones escalonadas de la "línea del cero porciento" en tales variables (véanse los cuadros 4.1 a 4.5 ) resaltan con menor claridad que en 1940. Sin embargo, se observan inflexiones en la mayoría de ellas en los 10000 habitantes; en forma menos patente en los 15000 habitantes (cuadros 4.1 y 4.2); y débilmente en los 5000 habitantes, como lo ilustran los cuadros 4.3 y 4.4. La escasa distinción que se manifiesta entre los intervalos $A$ y $B$ puede deberse en parte a no haberse incluido en el intervalo $A$ localidades menores de 2500 habitantes (véase la nota 31 ); también a que las localidades seleccionadas están cargadas hacia entidades más rurales - como se prueba posteriormente-, todo lo cual tiende a uniformar las características de los primeros dos intervalos.

Las características de las localidades estudiadas, tanto en 1940 como en 1960 (véase pp. 8-9), que exigen reservas en la comparación entre ambos años, sólo permiten elaborar dos conjeturas: 1) que la clasificación de cuatro intervalos evolucionó en el período considerado a otra de tres, con puntos de inflexión en los 10000 y 15000 habitan- 
tes; y 2) con base en el acelerado proceso de urbanización del país de 1940 a 1960 , que las características mixtas-rurales se están manifestando a partir de puntos anteriores a los 5000 habitantes, lo cual no es posible determinar en este estudio.

En síntesis, se concede mayor peso a las inferencias obtenidas del análisis de 1940, especialmente para los puntos 10000 y 15000 habitantes, por basarse en el estudio de todas las localidades de 10000 y más habitantes y además porque los datos censales sobre las mismas son a nivel de localidad. El análisis de 1960 confirma, pero a veces débilmente, la existencia de inflexiones en los puntos antes mencionados. ${ }^{32}$ La distinción en los 5000 habitantes es la que destaca menor número de veces y con menor claridad; sin embargo, se cree que esto se debe a la ubicación geográfica de las localidades estudiadas.

\section{OBSERVACIONES SOBRE LOS INTERVALOS DE LA CLASIFICACIÓN}

Las clasificaciones urbano-rurales más apegadas a la realidad socioeconómica de un país son las de índole multidimensional.33 En México, no es factible llegar a este tipo de solución, porque ello implica por necesidad un estudio de casos, y a la fecha no se dispone de datos suficientes a nivel de localidad para realizarlo.

Por lo tanto, teniendo en cuenta las limitaciones implícitas en una clasificación unidimensional como la obtenida, se procedió a la estimación del número de excepciones en cada uno de los intervalos de población, con objeto de apreciar con mayor detalle las desviaciones, tanto de la clasificación, como de la selección de localidades estudiadas.

Las localidades que manifiestan atributos distintos a los correspondientes a su clase de población se identificaron mediante un análisis comparativo de las variables dependientes en todas las localidades seleccionadas, de tal modo que:

a) subirían de clase las localidades que tuvieran más del $70 \%$ en dos o más variables (dos únicamente, si una era PEA agrícola), y

b) bajarían de clase las localidades que tuvieran menos del $30 \%$ en dos o más variables (dos únicamente, si una era PEA no agrícola).

32 Para medir la pérdida de información de esta clasificación con respecto a una dicotómica y otra formada de tres grupos de' localidades, se utilizó un índice basado en la "teoría de la información" $\left(I_{o} / I\right)$, que relaciona el contenido de información de una clasificación con grupos agregados $\left(I_{o}\right)$ con la correspondiente a la de cuatro intervalos $(I)$. Para detalles sobre el particular, véase $\mathrm{H}$. Theil y P. Uribe, The Information Approach to the Aggregation of Input-Output Tables (inédito). De la aplicación de tal índice a las variables $a$ ), b) y $d$ ) en 1940 , se encontró que una clasificación dicotómica en los 10000 habitantes arroja una pérdida de información cercana al $50 \%$. En cuanto a la clasificación de tres grupos (menos de $10000,10000-15000$ y más de 15000 habitantes), se obtuvo una pérdida de información del $4 \%$ en las variables mencionadas. De lo anterior se deduce que la clasificación de cuatro grupos contiene mayor información que las otras dos; y que la poca pérdida de información de la clasificación de tres intervalos se considera que está influida por la distorsión de la selección de localidades hasta los 10000 habitantes hacia entidades más rurales. (Véase la nota 35 .)

33 Duncan, op. cit., p. 45. 
Según estos criterios, la gráfica 1 indica el número y porciento de localidades que debieran cambiar de posición, tanto de los intervalos inferiores a los superiores como viceversa. Destaca el hecho de que son las clases extremas las que ganan localidades a base de las inter-

\section{Gráfica 1}

NÚMERO Y POR CIENTO DE LOCALIDADES QUE POR SUS CARACTERÍSTICAS PUEDEN CONSIDERARSE EN UNA CLASE DIFERENTE A SU INTERVALO DE POBLACIÓN

$\underline{1940}$

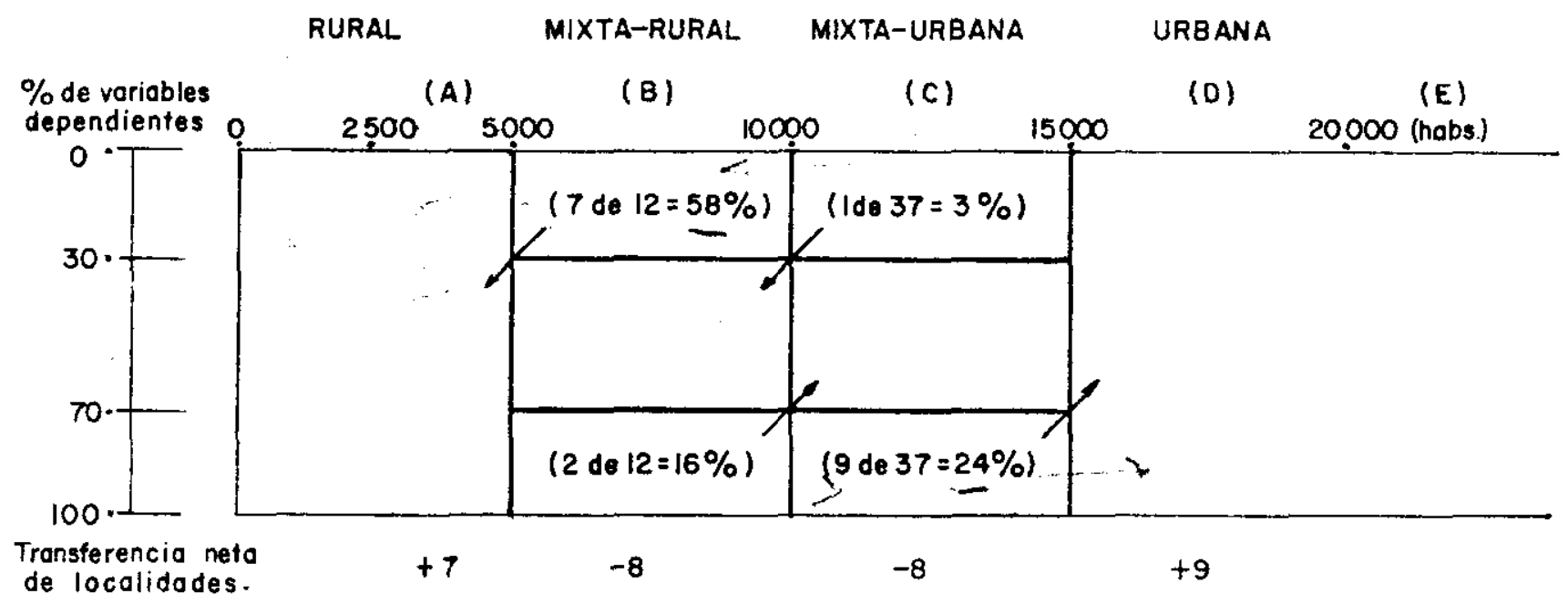

1960

RURAL MIXTA-RURAL MIXTA-UREANA URBANA

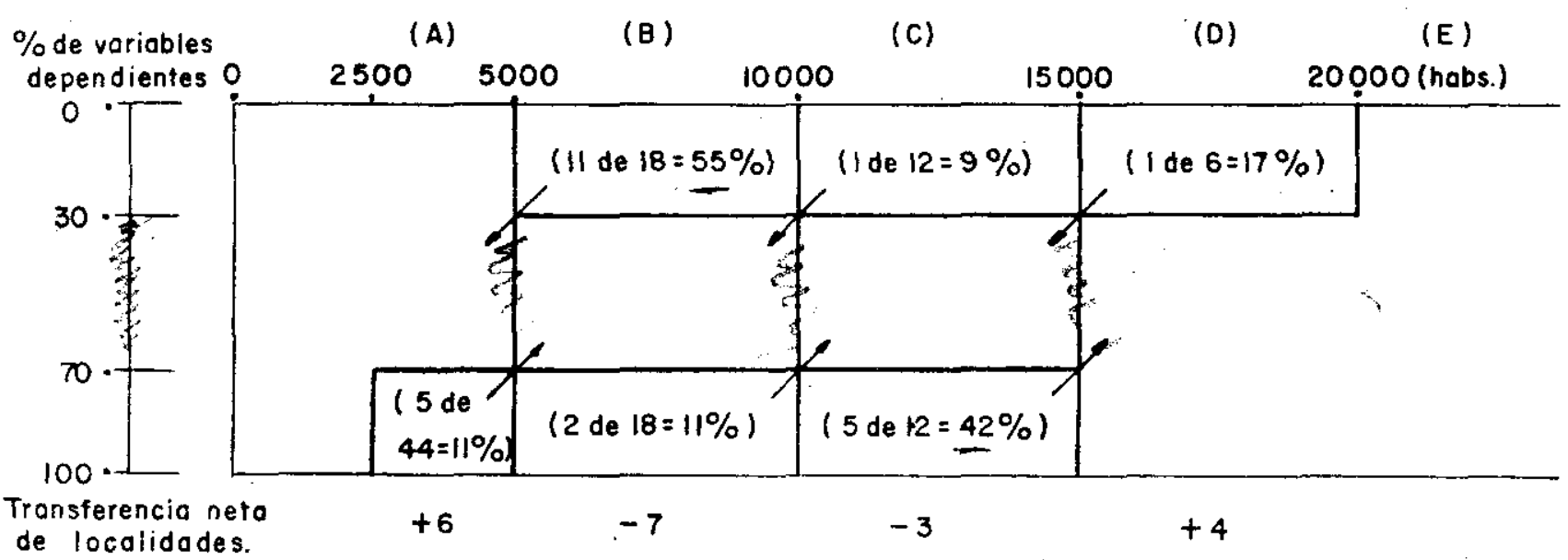

Nota: Las cifras entre paréntesis son el número y porciento de localidades que debieran corresponder a otra clase de localidades.

medias, lo que nos señala un mayor número de excepciones en estas últimas y por lo tanto su carácter transicional.

El elevado número de excepciones, principalmente en el grupo mixto-rural, puede explicarse por la distorsión que presenta la selección de localidades hacia entidades más rurales, lo cual tiende a homogeneizar las características de los intervalos $A$ y $B$, y por lo tanto dificulta la distinción de la división en los 5000 habitantes.

Los cuadros 1 y 2 muestran que la gran mayoría de las localidades 
estudiadas menores de 10000 habitantes pertenecen a entidades predominantemente rurales. ${ }^{34}$ Asimismo corresponde a tales entidades la gran mayoría de las localidades que descienden de clase, mientras que las que ascienden pertenecen a entidades más urbanizadas. ${ }^{\mathbf{3 5}}$

Una conclusión que se desprende del análisis anterior es la manifestación en México de diferencias regionales en las características de las localidades de menos de 10000 habitantes, ya que a igual tamaño de población, las localidades en las entidades más urbanizadas, como las de la frontera norte, registran altos porcientos en las variables socioeconómicas estudiadas, mientras que en las entidades más rurales predominan las localidades con porcientos más bajos.

\section{CONCLUSIONES}

1. Existe consenso general sobre las deficiencias de la definición censal de México, pues ésta no distingue debidamente la población urbana de la no urbana; presenta limitaciones para el-análisis de la realidad del país y deficiencias de orden teórico.

2. En México, las características de la población no son dicotómicas y sí manifiestan en algunos casos un claro continuum rural-urbano, lo cual se ajusta a los razonamientos teóricos.

3. La clasificación establecida, de cuatro intervalos de población, manifiesta mayor claridad y validez en el límite urbano-no urbano que en el límite rural-no rural.

4. Se considera aceptable el límite en los 5000 habitantes. Sin embargo, convendría ampliar el análisis en este extremo de la escala utilizando una selección de localidades más amplia y representativa.

5. Las diferencias regionales resultaron marcadas, especialmente en el extremo rural; menos destacadas fueron las diferencias en el tiempo.

6. A pesar de la heterogeneidad interna de los intervalos de la clasificación establecida, ésta se considera más representativa de la realidad urbano-rural que las clasificaciones censales y no censales aplicadas en el país, y constituye en consecuencia un instrumento de análisis útil para estudiar en forma más sistemática el proceso de urbanización en México.

34 Para identificar a las entidades predominantemente rurales en 1940, se analizó el índice indígena-colonial manejado por Whetten, y formado por la combinación de indicadores sobre lengua, vestido y calzado. En 1940, en aproximadamente el $66 \%$ de las entidades el índice resultó inferior al nacional tanto en localidades mayores como menores de los 10000 habitantes, y por lo tanto consideradas como más rurales. Las excepciones fueron las entidades de la frontera norte y Sinaloa, Quintana Roo, Aguascalientes, el Distrito Federal, Durango y Zacatecas. Ver Whetten, op. cit., cuadro 76, p. 364.

35 De las 8 y 13 localidades que descienden de intervalo en 1940 y 1960, respectivamente, todas corresponden a entidades predominantemente rurales. Por otro lado, de las 11 y 12 que ascienden de grupo en 1940 y 1960, pertenecen a entidades más rurales sólo 4 y 3 , respectivamente. 